\title{
Surgical Resection of Mitral Valve Papillary Fibroelastoma: A Robot-Assisted, Minimally Invasive Approach with Three-Dimensional Transesophageal Echocardiography Imaging
}

\author{
Crystal R. Bonnichsen ${ }^{1}$, Harold M. Burkhart ${ }^{2}$, Kyle W. Klarich ${ }^{1}$, Rakesh M. Suri ${ }^{2}$ \\ ${ }^{1}$ Division of Cardiovascular Diseases, Mayo Clinic, Rochester, USA \\ ${ }^{2}$ Division of Cardiovascular Surgery, Mayo Clinic, Rochester, USA \\ Email: suri.rakesh@mayo.edu
}

Received February 29, 2012; revised April 1, 2012; accepted April 28, 2012

\begin{abstract}
Papillary fibroelastomas (PFEs) are benign tumors of the endocardium that most frequently affect cardiac valves and typically present with embolic symptoms such as stroke or transient ischemic attack (TIA). Surgical excision is usually recommended for left-sided tumors and is associated with excellent long-term outcomes. The use of a robot-assisted, minimally invasive surgical approach for management of mitral valve disease is growing, and has been associated with shorter hospital stays and improved early quality of life. Three-dimensional (3D) transesophageal echocardiography (TEE) offers several advantages in the assessment of mitral valve disease and cardiac tumors, including the ability to precisely locate the site of attachment of the mass and the spatial relationships to surrounding structures. These factors are particularly important when planning a surgical approach. We report two cases of mitral valve PFEs which were successfully removed using a robot-assisted, minimally invasive surgical approach with 3D TEE imaging. This approach to treatment of PFEs is an attractive alternative to the traditional approach involving median sternotomy.
\end{abstract}

Keywords: Papillary Fibroelastoma; Robotic Surgery; Three-Dimensional Transesophageal Echocardiography

\section{Introduction}

Papillary fibroelastomas (PFEs) are benign tumors of the endocardium and are most commonly found on the leftsided cardiac valves, although they can occur on any endocardial surface. Presenting symptoms are usually embolic in nature, most commonly stroke or transient ischemic attack (TIA) [1]. Surgical excision results in excellent outcomes with no reported recurrences in the literature. We present two cases of patients with PFEs where a robot-assisted, minimally invasive surgical approach was utilized for excision of the fibroelastoma. Intraoperative three-dimensional transesophageal echocardiography (3D TEE) was utilized for pre-procedural imaging.

\section{Case Reports}

\subsection{Case 1}

A 49-year-old woman was referred to our institution for evaluation of a mass on the mitral valve. A stress echocardiogram had been performed to evaluate atypical chest pain and was negative for ischemia, but a broad-based soft tissue mass was noted on the left atrial aspect of the anterior leaflet of the mitral valve. There was concern for vegetation, although she had no history of fevers or systemic symptoms. Complete blood count and sedimentation rate were normal and blood cultures were negative. TEE was performed, anticoagulation with warfarin was initiated, and she was referred to our institution for further evaluation. She had no prior history of stroke or TIA, but did have one episode of transient blurred vision about four weeks prior to evaluation.

Transthoracic echocardiogram (TTE) was performed at our institution, revealing a 6 millimeter $(\mathrm{mm}) \times 6 \mathrm{~mm}$ round density on the anterior leaflet of the mitral valve suggestive of PFE (Figure 1) and concomitant mild mitral regurgitation. The left ventricular size and function were normal with an ejection fraction of $60 \%$. The TEE from the outside institution was reviewed and revealed a small, round density on the anterior leaflet of the mitral valve suggestive of PFE. The patient was evaluated by a cardiovascular surgeon and was deemed to be a candidate for a minimally invasive, robot-assisted approach.

Intra-operative TEE was performed and revealed a well-circumscribed mass attached to the anterior mitral 
leaflet (Figure 2). Three-dimensional imaging showed the tumor to be attached to the middle scallop of the anterior mitral leaflet (Figure 3). Following cannulation of the right femoral vessels for cardiopulmonary bypass, a $2.5 \mathrm{~cm}$ right thoracic access port was made and three ports for the robotic instruments were placed through the chest wall. The heart was arrested utilizing a transthoracic aortic crossclamp and antegrade cold cardioplegic solution. A left atriotomy was performed posterior to the interatrial groove to expose the anterior mitral lesion. The mass was sharply resected at its stalk-like base, leaving no residual tumor (Figure 4). Gross pathology revealed a papillary tumor measuring $6 \mathrm{~mm}$ with numerous delicate fronds, and microscopic evaluation with Verhoeff-Van Giesen (VVG) stain was consistent with PFE. The surgical procedure was uncomplicated: The patient was extubated in the operating room, transitioned to floor care the same night, and dismissed from hospital on postoperative day three.

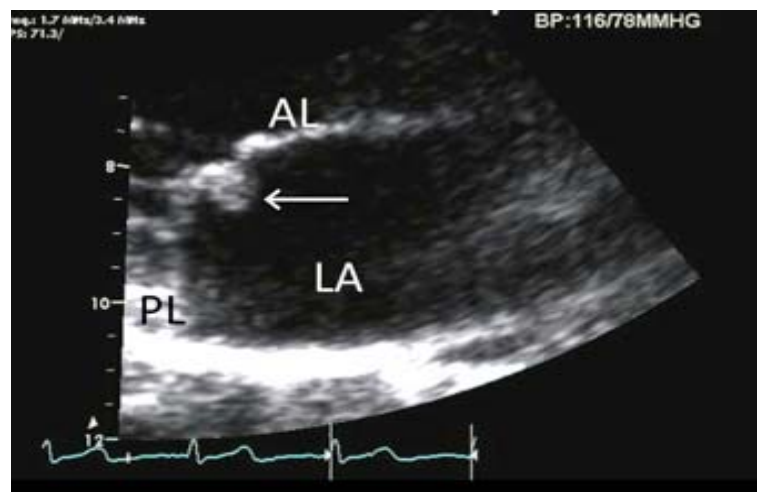

Figure 1. Transthoracic echo image from parasternal long axis reveals a well-circumscribed mass (arrow) attached to the anterior leaflet of the mitral valve, measuring $6 \mathbf{m m}$. There was no significant mitral regurgitation. $L A=$ left atrium; $A L=$ anterior leaflet; $P L=$ posterior leaflet.

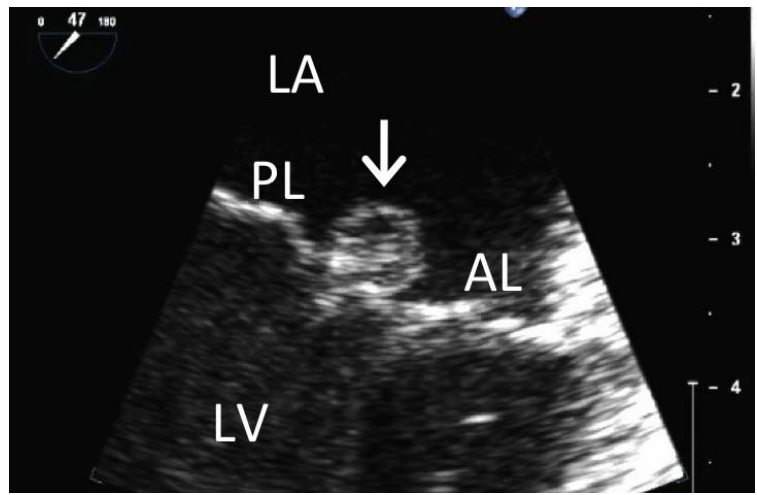

Figure 2. Intra-operative transesophageal echo (TEE) image of the mitral valve shows the well-circumscribed mass (arrow) attached to the anterior leaflet of the mitral valve. $\mathbf{L A}=$ left atrium; $\mathbf{L V}=$ left ventricle; $\mathbf{A L}$ = anterior leaflet; PL $=$ posterior leaflet.

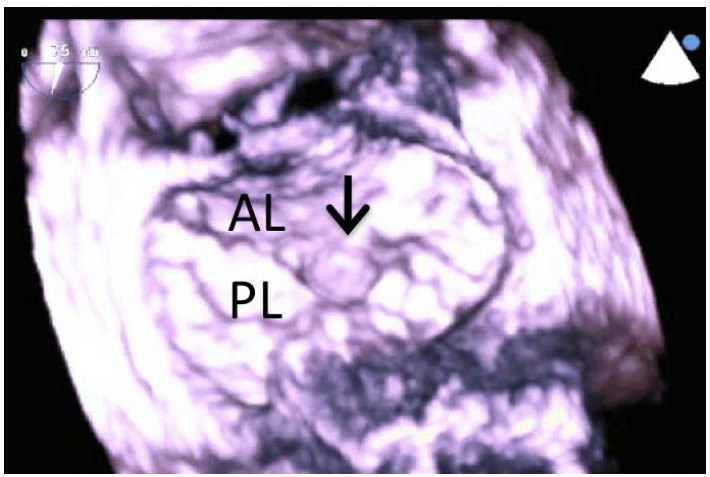

Figure 3. Still frame from three-dimensional (3D) TEE imaging reveals the mass (arrow) to be attached to the middle scallop of the anterior leaflet of the mitral valve. $A L=$ anterior leaflet; $\mathrm{PL}=$ posterior leaflet.

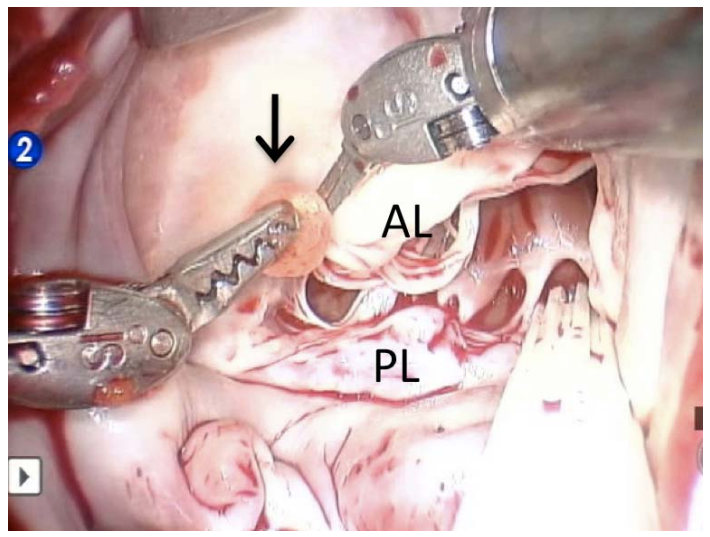

Figure 4. Still frame from intra-operative video as the mass (arrow) is removed from the anterior leaflet of the mitral valve. $\mathbf{A L}=$ anterior leaflet; $\mathbf{P L}=$ posterior leaflet.

\subsection{Case 2}

A 49-year-old man was referred to our institution for further evaluation of a mass on the posterior leaflet of the mitral valve. He presented to an outside facility for evaluation of recurrent TIAs, which included symptoms of right arm numbness, weakness, and tingling associated with slurred speech and tunnel vision. Magnetic resonance imaging (MRI) of the head revealed chronic areas of infarction in multiple vascular distributions, consistent with embolic events. A transthoracic echo was suggestive of a mass on the mitral valve and a subsequent TEE confirmed the presence of a mass near the posterior leaflet of the mitral valve.

TEE revealed a $9 \mathrm{~mm}$ spherical mass with features suggestive of PFE. The mass appeared to be attached to the chordae tendineae just beneath the posterior leaflet with no distinct attachment to the leaflet itself visualized (Figure 5). Live 3D imaging confirmed this finding (Figure 6). There was trivial mitral regurgitation. The left ventricular size was normal with an ejection fraction of $65 \%$. 


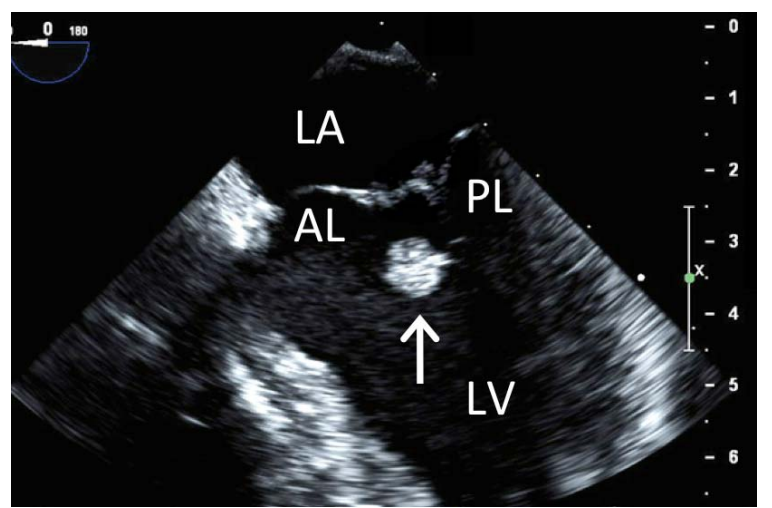

Figure 5. TEE shows a 9 mm well-circumscribed mass (arrow) which appears to be attached to the chordae tendineae just below the posterior leaflet of the mitral valve. No definite attachment to the leaflet is visualized. LA = left atrium; $\mathbf{L V}=$ left ventricle; $\mathbf{A L}=$ anterior leaflet; $\mathrm{PL}=$ posterior leaflet.

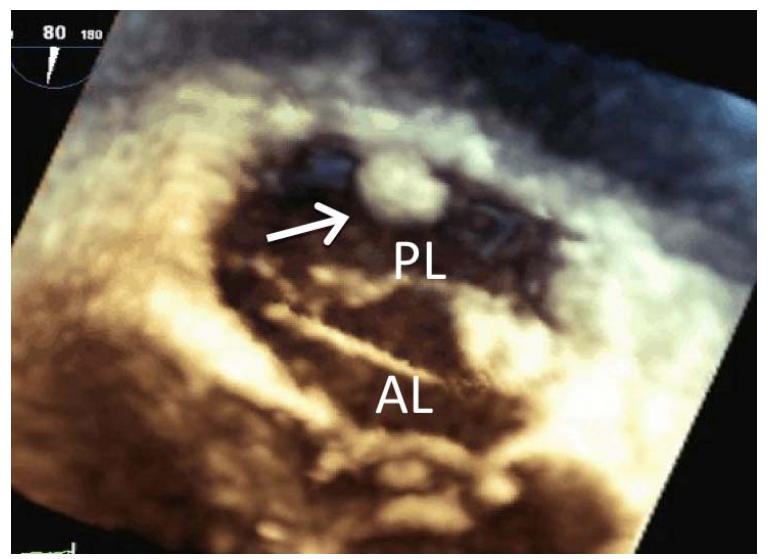

Figure 6. Still frame from 3D TEE imaging shows the mass (arrow) is not attached to the mitral leaflet and appears to be attached below the level of the valve. $\mathrm{AL}=$ anterior leaflet; $\mathrm{PL}=$ posterior leaflet.

The patient underwent robot-assisted, minimally invasive excision of a $9 \mathrm{~mm}$ PFE attached to the chordae arising from the posterolateral papillary muscle to the middle scallop of the posterior leaflet. Pathology was consistent with PFE, evaluated with VVG stain. The surgical procedure was uncomplicated, and the patient was dismissed on post-operative day four.

\section{Discussion}

PFEs are benign tumors of the endocardium, and are the most common tumor of the cardiac valves [2]. Grossly they consist of multiple frond-like projections and have been described as having a "sea anemone" appearance in water. Histologically, they consist of a central avascular collagenous core of dense connective tissue covered with hyperplastic endocardial cells. The left-sided valves are most commonly affected [1]. The average size is usually less than 1 centimeter $(\mathrm{cm})$, and they are typically pedunculated and exhibit independent mobility. On transthoracic echo they have been described as having a speckled appearance with echolucencies near the edges and which appear stippled and they appear to "shimmer" or "vibrate" at the tumor-blood interface [3].

PFEs are most frequently an incidental finding noted at the time of echocardiography. If symptoms are present, the most common presenting symptom is stroke or TIA if the location is the mitral valve, and MI or sudden death if the lesion arises from the aortic valve [1]. If patients have symptoms or if the tumor is located on the left side of the heart, surgical excision is usually recommended to prevent complications. A review of the surgical experience with PFEs from our institution [4] showed that surgical removal by shave excision alone was possible in $83 \%$ of 88 patients, while $9 \%$ required shave excision and valve repair. Five patients (6\%) required valve replacement; however two of those patients had concurrent degenerative valve disease. There were no recurrences of tumor, symptoms, or embolic events.

The use of minimally invasive surgery, specifically the use of robotic assistance, has increased over the past decade. This approach has been most commonly used for mitral valve repair, and has been associated with fewer blood transfusions, shorter hospital stay, and quicker return to preoperative level of functioning [5]. The specific benefits of a robotic approach for the removal of a PFE include the use of small port-access incisions to access intracardiac structures, 3D high definition videoscopic imaging of both atrial and ventricular structures, and the use of fine-tipped instruments to resect friable tumor material. The use of this approach for resection of intracardiac masses is limited to a few case reports. The use of robotic assistance was reported in a series of patients with cardiac myxomas [6] and was shown to be safe with good surgical outcomes. Woo and colleagues [7] reported the use of a minimally invasive approach utilizeing robotic assistance for removal of a PFE from the aortic valve.

3D TEE has been shown to add incremental value over two-dimensional echo in the evaluation of mitral valve disease. The major advantage of 3D TEE is the ability to be able to precisely locate the anatomic abnormality and mechanism of regurgitation, which is especially helpful during surgical repair [8-10]. There are multiple reports in the literature describing the use of 3D TEE [11-14] and 3D TTE [15-17] for evaluation of PFEs, with the major advantage being the ability to better understand the site of attachment and the spatial relationships with surrounding structures, which is helpful in planning a surgical approach.

The use of intraoperative 3D TEE for better visualization of the location of these masses and their sites of at- 
tachment coupled with a minimally invasive, robot-assisted surgical approach makes this an attractive approach for definitive treatment of PFEs. The combination of a minimally invasive approach, guided by 3D TEE, has the potential to facilitate the timely and accurate prophylactic removal of these potentially disastrous lesions, even in otherwise symptomatic patients.

\section{REFERENCES}

[1] R. M. Gowda, I. A. Khan, C. K. Nair, N. J. Mehta, B. C. Vasavada and T. J. Sacchi, "Cardiac Papillary Fibroelastoma: A Comprehensive Analysis of 725 Cases," American Heart Journal, Vol. 146, No. 3, 2003, pp. 404-410. doi:10.1016/S0002-8703(03)00249-7

[2] F. H. Edwards, D. Hale, A. Cohen, L. Thompson, A. T. Pezzella and R. Virmani, "Primary Cardiac Valve Tumors," Annals of Thoracic Surgery, Vol. 52, No. 5, 1991, pp. 1127-1131. doi:10.1016/0003-4975(91)91293-5

[3] K. W. Klarich, M. Enriquez-Sarano, G. M. Gura, W. D. Edwards, A. J. Tajik and J. B. Seward, "Papillary Fibroelastoma: Echocardiographic Characteristics for Diagnosis and Pathologic Correlation,” Journal of the American College of Cardiology, Vol. 30, No. 3, 1997, pp. 784-790. doi:10.1016/S0735-1097(97)00211-8

[4] D. L. Ngaage, C. J. Mullany, R. C. Daly, J. A. Dearani, W. D. Edwards, H. D. Tazelaar, C. G. McGregor, T. A. Orszulak, F. J. Puga, H. V. Schaff, T. M. Sundt III and K. J. Zehr, "Surgical Treatment of Cardiac Papillary Fibroelastoma: A Single Center Experience with Eighty-Eight Patients,” Annals of Thoracic Surgery, Vol. 80, No. 5, 2005, pp. 1712-1718. doi:10.1016/j.athoracsur.2005.04.030

[5] P. Modi, A. Hassan and W. R. Chitwood Jr., "Minimally Invasive Mitral Valve Surgery: A Systematic Review and Meta-Analysis," European Journal of Cardio-Thoracic Surgery, Vol. 34, No. 5, 2008, pp. 943-952. doi:10.1016/j.ejcts.2008.07.057

[6] C. Gao, M. Yang, G. Wang, J. Wang, C. Xiao, Y. Wu and J. Li, "Excision of Atrial Myxoma Using Robotic Technology," Journal of Thoracic and Cardiovascular Surgery, Vol. 143, Suppl. 4, 2009, pp. S64-S67.

[7] Y. J. Woo, T. J. Grand and S. J. Weiss, "Robotic Resection of an Aortic Valve Papillary Fibroelastoma," Annals of Thoracic Surgery, Vol. 80, No. 3, 2005, pp. 1100-1102. doi:10.1016/j.athoracsur.2004.02.108

[8] J. Grewal, S. Mankad, W. K. Freeman, R. L. Click, R. M. Suri, M. D. Abel, J. K. Oh, P. A. Pellikka, G. C. Nesbitt, I. Syed, S. L. Mulvagh and F. A. Miller, "Real-Time ThreeDimensional Transesophageal Echocardiography in the Intraoperative Assessment of Mitral Valve Disease,” Journal of the American Society of Echocardiography, Vol. 22, No. 1, 2009, pp. 34-41. doi:10.1016/j.echo.2008.11.008

[9] J. Manda, S. K. Kesanolla, M. C. Hsuing, N. C. Nanda, E. Abo-Salem, R. Dutta, C. A. Laney, J. Wei, C. Y. Chang, S. K. Tsai, S. Hansalia, W. H. Yin and M. S. Young,
"Comparison of Real Time Two-Dimensional with Live/ Real Time Three-Dimensional Transesophageal Echocardiography in the Evaluation of Mitral Valve Prolapse and Chordae Rupture,” Echocardiography, Vol. 25, No. 10, 2008, pp. 1131-1137. doi:10.1111/j.1540-8175.2008.00832.x

[10] R. Sharma, J. Mann, L. Drummond, S. A. Livesey and I. A. Simpson, "The Evaluation of Real-Time 3-Dimensional Transthoracic Echocardiography for the Preoperative Functional Assessment of Patients with Mitral Valve Prolapse: A Comparison with 2-Dimensional Transesophageal Echocardiography," Journal of the American Society of Echocardiography, Vol. 20, No. 8, 2007, pp. 934-940. doi:10.1016/j.echo.2007.01.028

[11] T. G. Kelpis, V. N. Ninios, V. A. Economopoulos and A. A. Pitsis, “Aortic Valve Papillary Fibroelastoma: A ThreeDimensional Transesophageal Echocardiographic Appearance,” Annals of Thoracic Surgery, Vol. 89, No. 6, 2010, p. 2043. doi:10.1016/j.athoracsur.2009.09.033

[12] S. Muller, G. Feuchtner, J. Bonatti, L. Muller, G. Laufer, R. Hiemetzberger, O. Pachinger, V. Barbieri and T. Bartel, "Value of Transesophageal 3D Echocardiography as an Adjunct to Conventional 2D Imaging in Preoperative Evaluation of Cardiac Masses,” Echocardiography, Vol. 25, No. 6, 2008, pp. 624-631. doi:10.1111/j.1540-8175.2008.00664.x.

[13] H. S. Dod, N. C. Nanda, A. D. Pacifico, T. S. Winokur, G. Baweja, B. S. Ravi, T. Htay, A. Fadel, G. Agarwal and S. K. Upendram, "Images in Geriatric Cardiology. Definitive Diagnosis of Aortic Valve Papillary Fibroelastoma by Three-Dimensional Transesophageal Echocardiography,” American Journal of Geriatric Cardiology, Vol. 12, No. 1, 2003, pp. 60-62. doi:10.1111/j.1076-7460.2003.01786.x

[14] S. Goonewardena, L. Sugeng, J. K. Min and R. Lang, "Cardiac Papillary Fibroelastoma-A Volatile Variant," Echocardiography, Vol. 22, No. 6, 2005, pp. 536-537. doi:10.1111/j.1540-8175.2005.40016.x

[15] T. Le Tourneau, S. Pouwels, B. Gal, A. Vincentelli, A. S. Polge, G. Fayad, S. Marechaux, P. V. Ennezat, B. Jegou and G. Deklunder, “Assessment of Papillary Fibroelastomas with Live Three-Dimensional Transthoracic Echocardiography,” Echocardiography, Vol. 25, No. 5, 2008, pp. 489-495. doi:10.1111/j.1540-8175.2008.00631.X

[16] A. Singh, A. P. Miller, N. C. Nanda, S. Rajdev, F. Mehmood and K. Duncan, "Papillary Fibroelastoma of the Pulmonary Valve: Assessment by Live/Real Time ThreeDimensional Transthoracic Echocardiography,” Echocardiography, Vol. 23, No. 10, 2006, pp. 880-883. doi:10.1111/j.1540-8175.2006.00336.X

[17] W. Dichtl, L. C. Muller, O. Pachinger, S. P. Schwarzacher and S. Muller, "Images in Cardiovascular Medicine. Improved Preoperative Assessment of Papillary Fibroelastoma by Dynamic Three-Dimensional Echocardiography," Circulation, Vol. 106, No. 10, 2002, p. 1300. doi:10.1161/01.CIR.0000029819.77219.4F 